\title{
Tamoxifen enhances therapeutic effects of gemcitabine on cholangiocarcinoma tumorigenesis
}

\author{
Gu Jing ${ }^{1}$, Kaiyu Yuan ${ }^{1}$, Amy N Turk , Nirag C Jhala ${ }^{1}$, Juan P Arnoletti ${ }^{2}$, Kui Zhang ${ }^{3}$, Jay M McDonald ${ }^{1,4}$ and \\ Yabing Chen ${ }^{1,4}$
}

Cholangiocarcinoma is a highly malignant tumor with limited therapeutic options. We have previously reported that tamoxifen (TMX) induces apoptosis of cholangiocarcinoma cells and reduces cholangiocarcinoma tumorigenesis in mice. In the present studies, we determined the effect of combination therapy of TMX and gemcitabine (GMT), another chemotherapeutical reagent for many cancers, on cholangiocarcinoma tumorigenesis and investigated the responsible mechanisms. GMT inhibited cell growth and induced apoptosis of cholangiocarcinoma cells in a concentrationdependent manner. TMX enhanced GMT-induced apoptosis of cholangiocarcinoma cells. Consistently, GMT (15 mg/kg) inhibited cholangiocarcinoma tumorigenesis in nude mice by $50 \%$. TMX $(15 \mathrm{mg} / \mathrm{kg})$ enhanced the inhibitory effect of GMT on tumorigenesis by $33 \%$. The inhibition of tumor growth correlated with enhanced apoptosis in tumor tissues. To elucidate the mechanisms underlying the additive effects of TMX on GMT-induced apoptosis, we determined the activation of caspases in cholangiocarcinoma cells exposed to GMT, TMX, or both. Activation of caspases 9 and 3, as well as cytochrome $\mathrm{c}$ release to the cytosol, was demonstrated in cells exposed to both reagents. In contrast, TMX activated caspase 2, whereas GMT had no effect. Inhibition of caspase 2 activation decreased TMX-, but not GMT-, induced activation of caspase 3 and apoptosis of cholangiocarcinoma cells. Similarly, activation of caspase 2 was found in tumors from TMX-treated mice, but not GMT-treated mice. Therefore, the enhanced effect of TMX on GMT-induced cholangiocarcinoma cell death is partially mediated by activation of caspase 2. TMX and GMT both induce apoptosis and inhibit cholangiocarcinoma tumorigenesis, which may be attributed to the activation of distinct apoptosis signals by TMX and GMT. Our studies provide in vivo evidence and molecular insight to support the use of TMX and GMT in combination as an effective therapy for cholangiocarcinoma.

Laboratory Investigation (2011) 91, 896-904; doi:10.1038/labinvest.2011.60; published online 4 April 2011

KEYWORDS: apoptosis; cholangiocarcinoma; gemcitabine; tamoxifen; tumor xenograft

Cholangiocarcinoma is a highly malignant neoplasm originating from the bile duct epithelial cells or cholangiocytes of the intra- and extrahepatic biliary system. ${ }^{1}$ The pathogenesis of cholangiocarcinoma is strongly associated with chronic biliary tract infection and inflammation, such as chronic viral hepatitis, hepatolithiasis, choledochal cysts, and primary sclerosing cholangitis. ${ }^{2,3}$ Prognosis of cholangiocarcinoma is generally poor with a 5 -year survival of $<5 \% .{ }^{4-6}$ Median survival for patients with intrahepatic cholangiocarcinoma is 18-30 months. ${ }^{6}$ Surgical resection of early stage tumors is the only effective therapy for long-term survival. ${ }^{7}$ In the last few years, the incidence and mortality from cholangiocarcinoma has increased, ${ }^{8}$ warranting an increasing need for effective therapeutic drugs and strategies to prevent and treat this lethal tumor.

Stimulation of apoptosis has been promoted as a potential therapy for many cancers, including cholangiocarcinoma. Apoptosis is an important process in a wide variety of different biological systems, including normal cell turnover, development of the immune system, embryonic development, metamorphosis and hormone-dependent atrophy, and also in chemical-induced cell death. ${ }^{9}$ Many cancer cells show a decreased ability to undergo apoptosis in response to various stimuli, which results from either a deficiency of

${ }^{1}$ Department of Pathology, University of Alabama at Birmingham, Birmingham, AL, USA; ${ }^{2}$ Department of Surgery, University of Alabama at Birmingham, Birmingham, AL, USA; ${ }^{3}$ Department of Biostatistics, University of Alabama at Birmingham, Birmingham, AL, USA and ${ }^{4}$ Veterans Administration Medical Center, Research Department, Birmingham, AL, USA

Correspondence: Dr JM McDonald, MD, LHRB509, 701 19th ST S, Birmingham, AL 35294, USA and Dr Y Chen, PhD, Department of Pathology, University of Alabama at Birmingham, SHEL710, 1825 University Boulevard, Birmingham, AL 35294, USA.

E-mails: mcdonald@uab.edu and ybchen@uab.edu

Received 2 November 2010; revised 15 December 2010; accepted 23 December 2010 
proapoptotic molecules or expression of inhibitors of apoptotic pathways. ${ }^{10}$ Results from our group and others have suggested that regulation of Fas-mediated apoptosis is a promising therapeutic avenue for cholangiocarcinoma. ${ }^{10,11-16}$ We have demonstrated that a number of molecules significantly enhance Fas-mediated apoptosis in cholangiocarcinoma through different molecular mechanisms. ${ }^{10-14,16}$ In search of an efficient chemotherapy for cholangiocarcinoma, we identified that tamoxifen (TMX), a potent calmodulin antagonist, induces apoptosis in cholangiocarcinoma cells in vitro and inhibits tumorigenesis in vivo in nude mice xenografts. ${ }^{13}$ TMX is one of the most widely used anticancer drugs. It is inexpensive and has a low-side-effect profile, thus providing a very attractive therapeutic option. ${ }^{17,18}$ Although the primary mechanism of action of TMX is believed to be through the inhibition of the estrogen receptor (ER), research over the years has indicated that additional, non-ER-mediated mechanisms exist. These include modulation of signaling proteins such as protein kinase C, calmodulin, TGF- $\beta$, c-myc, MAPK, JNK, and p38. Oxidative stress, mitochondrial permeability transition, ceramide generation, as well as changes in cell membrane fluidity, may also have important roles in TMX-induced apoptosis. ${ }^{19}$ In cholangiocarcinoma, we found that TMXinduced apoptosis is partially dependent on activation of caspases, inhibition of pAKT, and FLIP expression. ${ }^{11}$ These studies have supported the use of TMX as a therapy for cholangiocarcinoma and possibly other malignancies that are ER negative. ${ }^{10-13}$

Gemcitabine (GMT) is used currently to treat cholangiocarcinoma and GMT-based combination regimens appear to improve the survival. ${ }^{6,20-25}$ GMT is a nucleoside analog. It acts directly by inhibiting DNA synthesis and also causes apoptosis. ${ }^{26,27}$ Mechanisms of GMT-mediated apoptosis have been investigated in several cancers. ${ }^{28-31}$ GMT has been increasingly used to treat hepatobiliary cancers because of its reasonable efficacy for treating pancreatic cancer. ${ }^{32}$ Low dose of GMT is preferred to avoid severe toxicity, especially pulmonary toxicity. ${ }^{33-36}$ Hence, combination therapies with GMT plus a variety of chemotherapeutic agents have been employed to minimize toxicity and maximize therapeutic efficacy. Currently, GMT combined with chemicals like leucovorin, 5-fluorouracil, capecitabine, and platinum agents have shown enhanced therapeutic effects in cholangiocarcinoma. ${ }^{6,20-25}$

Based on previously published data, TMX and GMT appear to induce apoptosis via distinct mechanisms. ${ }^{11,13,28-31}$ Therefore, we evaluated the efficacy of combination therapy with TMX and GMT on human cholangiocarcinoma tumorigenesis in a mouse xenograft model and investigated the responsible molecular mechanisms. We have found that TMX and GMT induce apoptosis and inhibit cholangiocarcinoma tumorigenesis. These effects were attributed to activation of distinct apoptosis signals by TMX and GMT. Our findings provide in vivo evidence and mechanistic molecular insight to support the use of TMX and GMT as an effective combination therapy for cholangiocarcinoma.

\section{MATERIALS AND METHODS Cell Culture, Antibodies and Reagents}

The cholangiocarcinoma cell line Sk-ChA-1 was originally provided by Dr A Knuth (Ludwig Institute for Cancer Research, London, UK). Cells were grown in RPMI 1640 (Invitrogen) supplemented with penicillin ( 5 units $/ \mathrm{ml}$ ), streptomycin $(5 \mu \mathrm{g} / \mathrm{ml})$, and $10 \%$ heat-inactivated fetal bovine serum. Cholangiocarcinoma cell line MZ-ChA-1 was kindly provided by Dr Gregory J Gores (Mayo Clinic, Rochester, MN, USA) and maintained as previously described. ${ }^{11,37}$ Antibodies to caspases 8 and 9 were purchased from BD Bioscience. Anti-caspase 2 antibody was obtained from Alexis Biochemicals. The caspase 3 antibody was from StressGen and the cytochrome c antibody was from PharMingen. Antibodies to PARP, GAPDH, and the caspase 2 inhibitor were purchased from Sant Cruz Biotech. Antiphospho-Histone $\mathrm{H} 3$ antibody was from Millipore. TMX was obtained from Sigma and GMT was purchased from Eli Lilly.

\section{Cell Survival Assay}

Cells were grown in 96-well plates at $4 \times 10^{3}$ cells per well and treated with $20 \mu \mathrm{M}$ TMX and/or $3 \mu \mathrm{M}$ GMT for $72 \mathrm{~h}$. After incubation, $20 \mu \mathrm{l}$ of MTS solution (Promega) were added into each well. The plate was incubated for $2 \mathrm{~h}$ at $37^{\circ} \mathrm{C}$. The absorbance at $570 \mathrm{~nm}$ was recorded with the microplate reader (BioTek).

\section{Assessment of Apoptosis}

Cholangiocarcinoma cells were exposed to a series of concentrations of GMT or $20 \mu \mathrm{M}$ TMX and/or $3 \mu \mathrm{M}$ GMT for $24 \mathrm{~h}$. Apoptosis was determined by Annexin V-FITC and propidium iodide staining (BD Biosciences) and analyzed by flow cytometry (BD Biosciences). The effect of caspase 2 inhibitor was determined with cells pretreated with caspase 2 inhibitor $(7.2 \mu \mathrm{M})$ for $2 \mathrm{~h}$ before addition of TMX or GMT.

\section{Cell Proliferation Assay}

Cells were grown in 96-well plates at $4 \times 10^{3}$ cells per well and treated with $20 \mu \mathrm{M}$ TMX and/or $3 \mu \mathrm{M}$ GMT for $48 \mathrm{~h}$. After incubation with $\mathrm{BrdU}$ for $2 \mathrm{~h}$ at $37^{\circ} \mathrm{C}$, cell proliferation was determined with a BrdU Cell Proliferation Assay kit (Calbiochem) following the manufacturer's protocol.

\section{Preparation of Cytosolic Fractions}

To extract cytosolic proteins, cells were washed twice with ice-cold PBS and resuspended in $500 \mu$ l homogenization buffer (20 mM HEPES, pH 7.4, $10 \mathrm{mM} \mathrm{KCl}, 1 \mathrm{mM} \mathrm{MgCl}_{2}$, $0.5 \mathrm{mM}$ EDTA, $1 \mathrm{mM}$ DTT, $250 \mathrm{mM}$ sucrose, and protease inhibitor cocktail tablets). After incubation on ice for $20 \mathrm{~min}$, cells were homogenized with a Dounce homogenizer and centrifuged at $1000 \mathrm{~g}$ for $10 \mathrm{~min}$ to separate nuclei and unbroken cells. The supernatants were centrifuged at $22000 \mathrm{~g}$ at 
$4{ }^{\circ} \mathrm{C}$ for 15 min to pellet membranes including mitochondria. The resulting supernatants were used as cytosolic extracts.

\section{Western Blot Analysis}

Protein extracts from cells were prepared as described previously. ${ }^{10,11}$ Concentrations of protein were determined with a BCA protein assay kit (Thermo Scientific). Proteins were separated by SDS-PAGE and transferred to Immobilon $\mathrm{P}$ membranes (Millipore) as described previously. ${ }^{10,11} \mathrm{Mem}$ branes were blocked in 5\% non-fat milk and incubated with primary antibodies overnight at $4{ }^{\circ} \mathrm{C}$. Horseradish peroxidase-conjugated secondary antibodies in the blocking buffer were incubated for $1 \mathrm{~h}$ at room temperature. Signals were detected using Immobilon Western chemiluminescent horseradish peroxidase substrate detection kit (Millipore).

\section{Mouse Xenograft Model}

The animal protocol was approved by the Institutional Animal Care and Use Committee at the University of Alabama at Birmingham, Birmingham, AL, USA. Male athymic nu/nu mice (4 weeks, NCI-Frederick) were used for tumor inoculation. Briefly, cholangiocarcinoma cells, SK-ChA-1 $\left(2 \times 10^{6}\right.$ cells in $200 \mu \mathrm{l} \mathrm{PBS} /$ site $)$, were inoculated subcutaneously into the flank area of mice. Mice bearing human cholangiocarcinoma xenograft tumors were randomly divided into three treatment groups and a control group (six mice per group) and treated with intraperitoneal injection of TMX $(15 \mathrm{mg} / \mathrm{kg}, 2$ consecutive days with 1 day rest), GMT $(15 \mathrm{mg} / \mathrm{kg}$, every third day), or the combination $(\mathrm{TMX}+\mathrm{GMT})$. The control group was injected with $0.9 \%$ sodium chloride. Tumor size and body weight were measured every 3 days and volumes were determined using the formula volume $=$ length $\times$ width $^{2} / 2$.

\section{TUNEL and Immunohistochemical Staining}

At the end of the experiment, tumors were removed. Half of each tumor was homogenized for western blot analysis. The other half was fixed in $4 \%$ paraformaldehyde and embedded in paraffin. Consecutive tumor sections $(8 \mu \mathrm{m})$ from each group were used for histological and immunohistochemial staining. To assess apoptotic cells in tumor tissues, TUNEL staining (DeadEnd Fluorometric TUNEL System; Promega) was performed following the manufacturer's protocol. For quantitative analysis, cell numbers were counted under the microscope $(\times 400)$. Four fields in each slide were counted and the percentage of apoptotic cells was determined. For the immunohistochemical analysis, slides were deparaffinized, rehydrated, and then heated in $10 \mathrm{mM}$ citrate buffer ( $\mathrm{pH}$ 6.0) for $40 \mathrm{~min}$ using a steamer. After washing in Tris-buffered saline, endogenous peroxidase activity was quenched by incubating the slides in 3\% hydrogen peroxide. The slides were blocked with 5\% FBS for $1 \mathrm{~h}$ at RT and incubated with the primary antibody $(1: 50)$ at $4{ }^{\circ} \mathrm{C}$ overnight. Then slides were treated with anti-rabbit horseradish peroxidase-labeled secondary antibody for $1 \mathrm{~h}$, developed using diaminobenzi- dine chromogen solution and counterstained with Mayer's hematoxylin. Brown staining in $>10 \%$ of the cells was considered as a positive stain.

\section{Statistical Analysis}

Results are expressed as mean \pm s.e. Differences between two groups were identified with Student's $t$-test. Significance was defined as $P<0.05$.

\section{RESULTS}

\section{TMX Enhances GMT-Induced Growth Inhibition and} Apoptosis in Cholangiocarcinoma Cells

To determine the effects of TMX and GMT on cholangiocarcinoma cell growth, the MTS assay was performed after exposure of Sk-ChA- 1 cells to $20 \mu \mathrm{M}$ TMX, $3 \mu \mathrm{M}$ GMT, or a combination of both for $72 \mathrm{~h}$. Compared with control, both TMX and GMT inhibit tumor cell growth (Figure 1a). The combination of TMX and GMT demonstrated slightly enhanced inhibition compared with each agent alone. We have reported that TMX induces apoptosis in human cholangiocarcinoma cells. ${ }^{11,12}$ To test whether the inhibition of cholangiocarcinoma cell growth by GMT was due to apoptosis, cholangiocarcinoma cells were exposed to a series of concentrations of GMT and apoptosis was determined $24 \mathrm{~h}$ after the incubation. As shown in Figure 1b, GMT induces apoptosis in Sk-ChA-1 cells in a dose-dependent manner. Furthermore, TMX was found to enhance the GMT-induced apoptosis in the Sk-ChA-1 cells (Figure 1c). Consistently, TMX enhanced GMT-induced apoptosis in an additional cholangiocarcinoma cell line, MZ-ChA-1 ${ }^{11,37}$ (Figure 1d).

\section{TMX Enhances the Inhibitory Effect of GMT on Cholangiocarcinoma Tumorigenesis in Mice}

The enhanced effects of the combination TMX and GMT over either agent alone in vitro suggested that TMX and GMT in combination would be an effective therapy for cholangiocarcinoma. To determine their efficacy in vivo, a cholangiocarcinoma xenograft model was employed. Male athymic nude mice bearing cholangiocarcinoma xenografts were treated with an intraperitoneal injection of TMX $(15 \mathrm{mg} / \mathrm{kg}$, 2 consecutive days with 1 day rest), GMT $(15 \mathrm{mg} / \mathrm{kg}$, every third day), or a combination of both for 21 days. Compared with controls, both TMX and GMT significantly inhibit tumor growth (TMX by $44 \%$ and GMT by $50 \%$ ) (Figure $2 \mathrm{a}$ and $b$ ). As expected, the combination of TMX and GMT was more effective than either agent alone. TMX enhanced the inhibitory effect of GMT on tumorigenesis by 33\% (Figure 2b). With current doses of TMX and GMT, no significant body weight loss was observed during the experiment (Figure 2c).

\section{TMX Enhances GMT-Induced Apoptosis in Cholangiocarcinoma Tumors}

Our in vitro data demonstrated that TMX enhances GMTinduced apoptosis in cholangiocarcinoma cells (Figure 1). In 


\section{a}
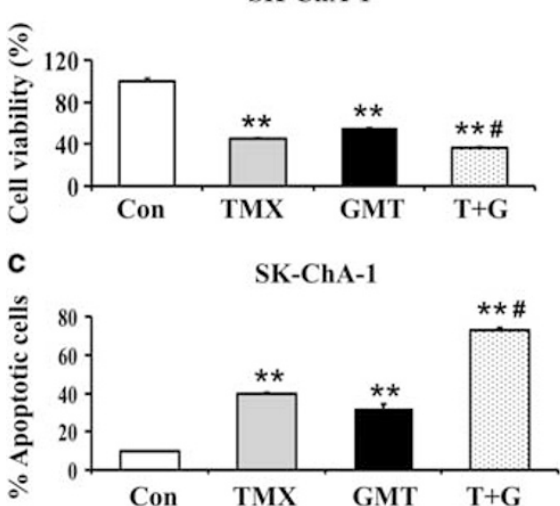
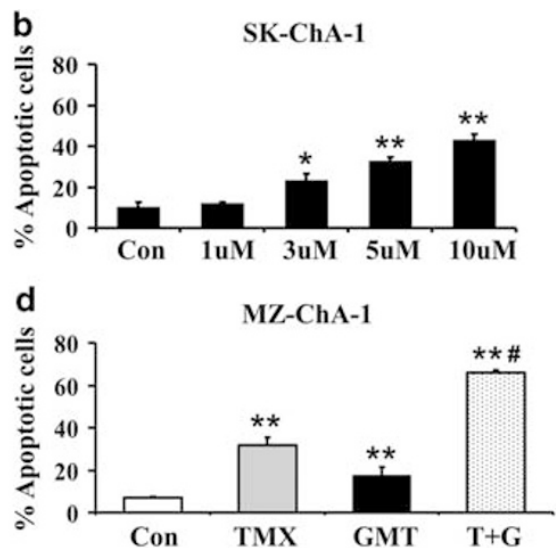

Figure 1 TMX enhances GMT-induced growth inhibition and apoptosis in cholangiocarcinoma cells. (a) Cholangiocarcinoma cells, SK-ChA-1, were exposed to $20 \mu \mathrm{M}$ TMX, $3 \mu \mathrm{M}$ GMT, or a combination of both for $72 \mathrm{~h}$. MTS assay was performed to detect the cell viability. Cholangiocarcinoma cells, SK-ChA-1 or MZ-ChA-1, were incubated with a series of concentrations of GMT (b) or $20 \mu \mathrm{M} \mathrm{TMX}$ and/or $3 \mu \mathrm{M}$ GMT (c, SK-ChA-1 cells; d, MZ-ChA-1 cells) for $24 \mathrm{~h}$ and then apoptosis was analyzed by Annexin V/propidium iodide staining. Results shown are mean $\pm \mathrm{s}$.e. $(n=3)$. ${ }^{\star} P<0.05$ and ${ }^{\star} * P<0.01$ for comparison with controls. ${ }^{\#} P<0.05$ for comparison with each agent alone.
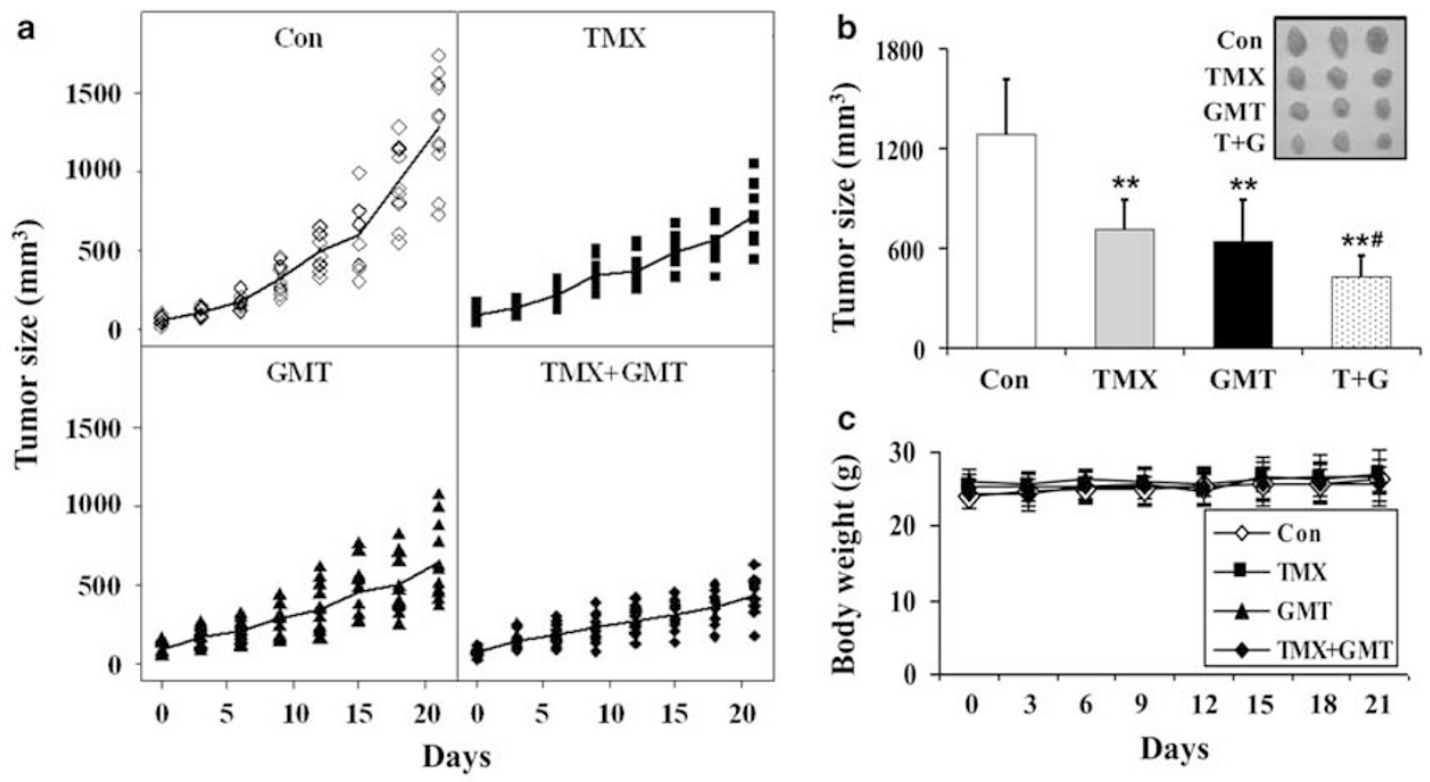

Figure 2 TMX enhances the inhibitory effect of GMT on cholangiocarcinoma tumorigenesis in mice. Cholangiocarcinoma cells, SK-ChA-1, were inoculated subcutaneously into the flank area of 4-week-old male athymic nude mice. After 5 days, mice were randomly assigned into four groups (six mice per group) and treated with intraperitoneal injection of TMX (15 mg/kg, 2 consecutive days with 1 day rest), GMT (15 mg/kg, every third day), or the combination (TMX + GMT). The control group was injected with $0.9 \%$ sodium chloride. Tumor size and body weight were measured every 3 days for 21 days. Tumor volumes were determined using the formula volume $=$ length $\times$ width $^{2} / 2$. (a) Average tumor volumes at different days in mice treated with $\mathrm{TMX}, \mathrm{GMT}$, or the combination. (b) Average tumor volumes at the end of experiments. Representative tumors were removed and photographed. (c) Body weights of mice in each group are also shown. Results shown are mean \pm s.e. ( $n=12$ for tumors and $n=6$ for body weights). ${ }^{\star *} P<0.01$ for comparison with controls and ${ }^{\#} P<0.05$ for comparison of each agent alone with combination therapy.

the in vivo studies, to determine whether the tumorigenesis inhibition was accompanied by enhanced apoptosis and whether GMT-induced apoptosis was enhanced by TMX in tumors, apoptotic cells in the tumor tissues were analyzed with TUNEL staining. As shown in Figure $3 a$ and $b$, TUNEL positive cells were enhanced in both TMX- and GMT-treated tumors compared with controls. Consistently, TMX was also found to enhance GMT-induced apoptosis in cholangiocarcinoma tumors (Figure 3a and b). Additionally, apoptosis in tumor tissues was confirmed by immunohistochemical staining of cleaved caspase 3 (Figure $3 a$ and c). Similarly, significantly increased cleaved caspase 3 positive cells were observed in tumors with combination therapy compared with either alone. Both results of TUNEL and immunohistochemical staining suggested that the inhibition of tumorigenesis was at least partially attributed to enhanced apoptosis for both TMX and GMT and for the combination of TMX and GMT. 
a
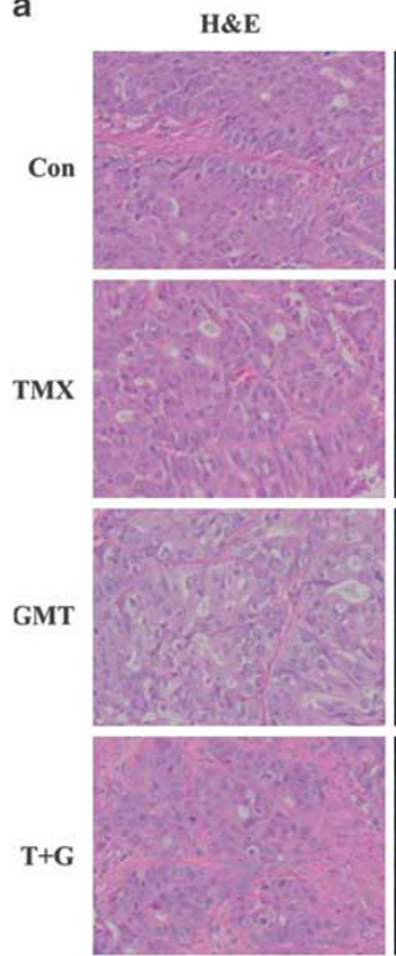

TUNEL
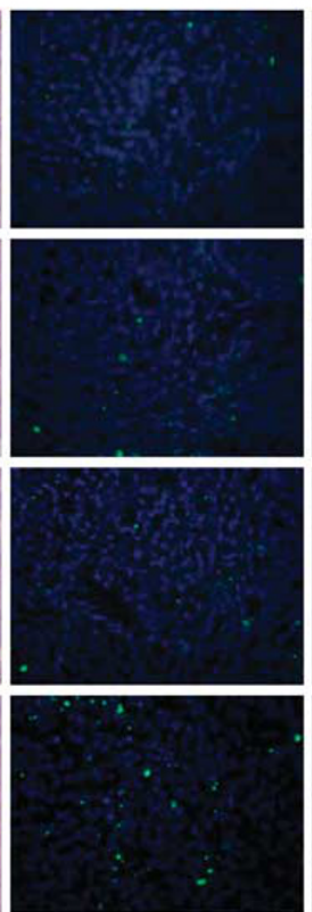
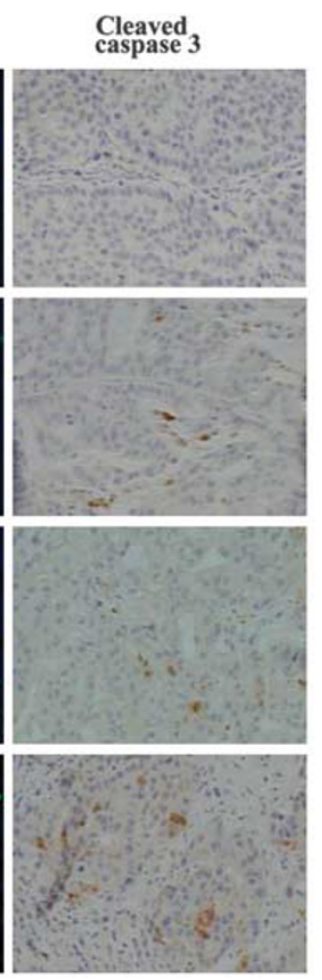

b
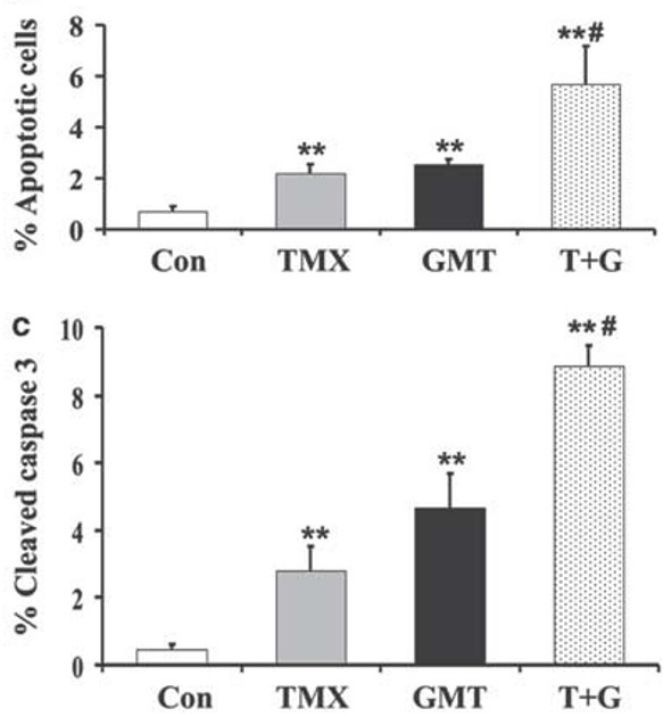

Figure 3 TMX enhances GMT-induced apoptosis in cholangiocarcinoma tumors. At the end of tumorigenesis experiment, tumors were processed as described in Materials and methods. Consecutive sections of tumors from each mouse were analyzed by (a) H\&E staining (first column), TUNEL staining (middle column), and immunohistochemical staining of cleaved caspase 3 (right column). Representative images of each group are shown. For quantitative analysis, cell numbers were counted and the percentage of positive cells was determined. (b) Percentage of apoptotic cells determined by TUNEL staining. (c) Percentage of cleaved caspase 3 stained cells. Results shown are mean \pm s.e. $(n=12)$. ${ }^{\star * P}<0.01$ for comparison with controls and ${ }^{\#} P<0.05$ for comparison of each agent alone with combination therapy.

\section{TMX and GMT Induce Activation of Different Apoptotic Signals in Cholangiocarcinoma Cells and in Cholangiocarcinoma Tumors in Mice}

To elucidate the mechanisms underlying the enhancement by TMX of GMT-induced apoptosis, we determined expression/ activation of caspases in cholangiocarcinoma cells stimulated by $20 \mu \mathrm{M}$ TMX, $3 \mu \mathrm{M}$ GMT, or a combination of both for 24 h. As shown in Figure 4a, caspase 3, a downstream caspase, was activated by both TMX and GMT, which was consistent with the apoptosis data (Figure 1c). Cleaved caspase 3 significantly increased after combined treatment with TMX and GMT, which supports our data that induction of apoptosis by TMX and GMT was enhanced over each agent alone (Figure 4a). To determine whether extrinsic (death receptor) or intrinsic (mitochondrial) pathways were activated by TMX or GMT, upstream caspases were checked. Activation of caspase 9, as well as cytoplasmic cytochrome c, were detected in cells exposed to both reagents (Figure $4 \mathrm{a}$ and $\mathrm{b}$ ). These results suggested that both TMX and GMT could act through the mitochondrial pathway. Interestingly, compared with GMT, the effects of TMX on cytochrome $c$ release and caspase 9 activation were much weaker (Figure 4a). However, their effects on the downstream caspase, caspase 3, were almost similar (Figure 4a). It appears that besides the mitochondrial pathway TMX may act through another signaling pathway leading to caspase 3 cleavage and apoptosis. To check this hypothesis, more upstream caspases were analyzed including caspase 8 involved in extrinsic pathway and caspase 2, which is multifunctional and has been demonstrated to act in parallel with both the mitochondrial and death receptor pathways of apoptosis. ${ }^{38-40}$ As shown in Figure 4a, TMX significantly activated caspase 2, whereas GMT had no effect. Therefore, TMX activates caspase 3 and induces apoptosis at least in part through activating caspase 2. In contrast, GMT predominantly activates caspases 9 and 8 .

To determine whether the mechanisms whereby TMX and GMT induces apoptosis in vivo are similar to these in vitro, tumor homogenates were analyzed with western blot analysis. As shown in Figure 4c, caspases 9 and 3 were activated by both TMX and GMT. Consistent with the observation with cholangiocarcinoma cells (Figure 4a), activation of caspase 2 was found in tumors from TMX-treated mice, but not GMTtreated mice. Therefore, in vitro and in vivo, the ability of TMX to enhance GMT-induced apoptosis and inhibit tumorigenesis was due at least in part to their activation of different apoptosis signals. 

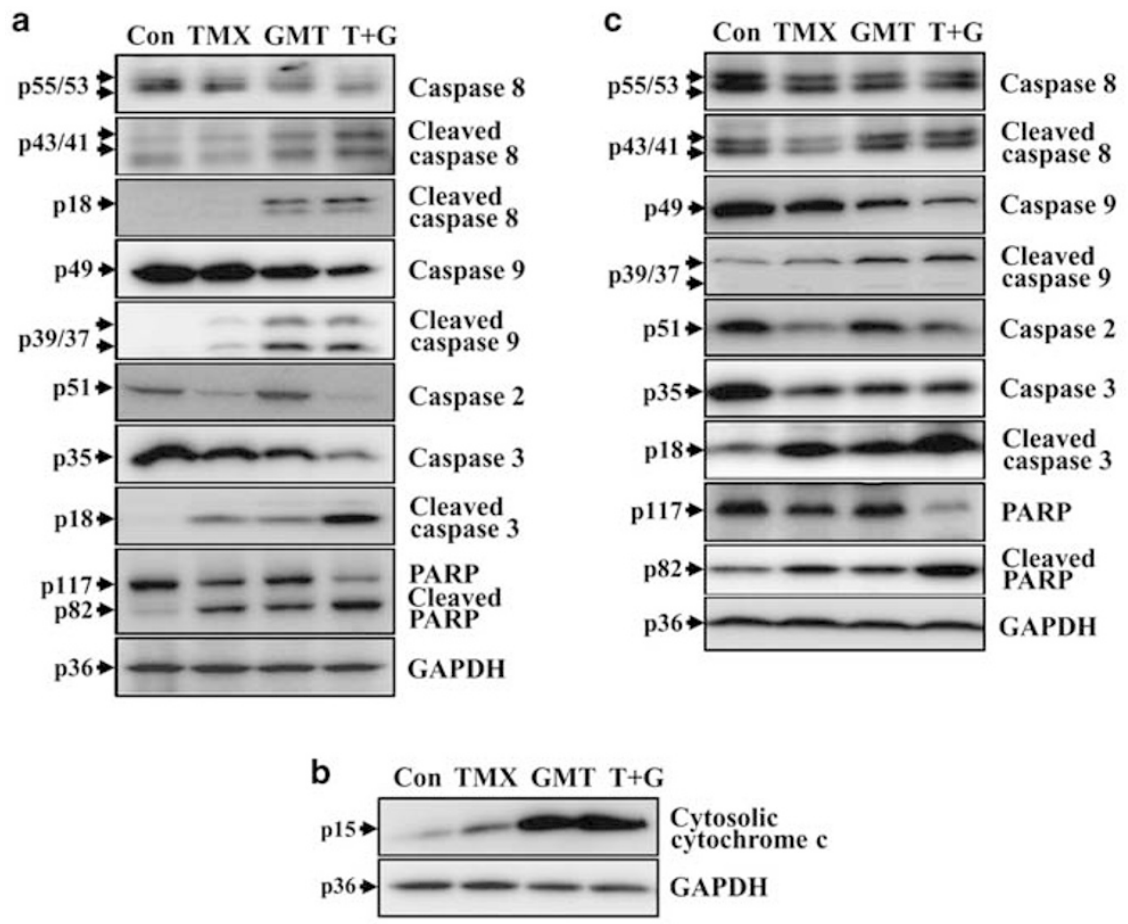

Figure 4 TMX and GMT induce activation of different apoptotic signals in cholangiocarcinoma cells and in cholangiocarcinoma tumors in mice. Cholangiocarcinoma cells, SK-ChA-1, were exposed to $20 \mu \mathrm{M} \mathrm{TMX}, 3 \mu \mathrm{M}$ GMT, or the combination for $24 \mathrm{~h}$. Western blot analysis was performed to determine (a) the expression/activation of caspases in whole cell lysates and (b) cytochrome $\mathbf{c}$ in the cytosolic fractions. (c) At the end of the animal experiment, tumor tissues were removed and half of each tumor was homogenized. The expression/activation of caspases in tumor homogenates was determined by western blot analysis.

\section{TMX-Induced Activation of Caspase 2 Contributes to Its Apoptotic Effects in Cholangiocarcinoma Cells}

To further confirm the requirement of caspase 2 for TMXinduced apoptosis, cholangiocarcinoma cells were preincubated with a caspase 2 inhibitor for $2 \mathrm{~h}$ and subsequencially exposed to TMX $(20 \mu \mathrm{M})$ or GMT $(3 \mu \mathrm{M})$ for $24 \mathrm{~h}$. Inhibition of caspase 2 activation significantly decreased TMX-, but not GMT-, induced activation of caspase 3 and apoptosis in cholangiocarcinoma cells (Figure 5). These data confirm that enhanced effect of TMX on GMT-induced apoptosis is mediated at least in part by activation of caspase 2 .

\section{DISCUSSION}

This study was designed to evaluate the efficacy and mechanism of combination therapy with TMX and GMT in cells and tumorigenesis models of human cholangiocarcinoma. Cholangiocarcinoma is a highly malignant tumor that arises from the bile ducts near or in the liver. ${ }^{11,41}$ It has a poor 5 -year survival rate and has no effective therapy. ${ }^{4-6}$ GMT is currently the leading therapy for cholangiocarcinoma. ${ }^{6,20-25}$ GMT directly alters DNA synthesis, causes DNA damage, and primarily triggers apoptosis by release of cytochrome $\mathrm{c}$ from the mitochondria. ${ }^{26-28}$ To avoid severe toxicity and improve survival, GMT-based therapy is often used in combination with other agents. ${ }^{6,20-25,33-36}$ We have previously shown that TMX, as a CaM antagonist, induces apoptosis in human cholangiocarcinoma cells, which lack ERs. ${ }^{10-13}$ We reported
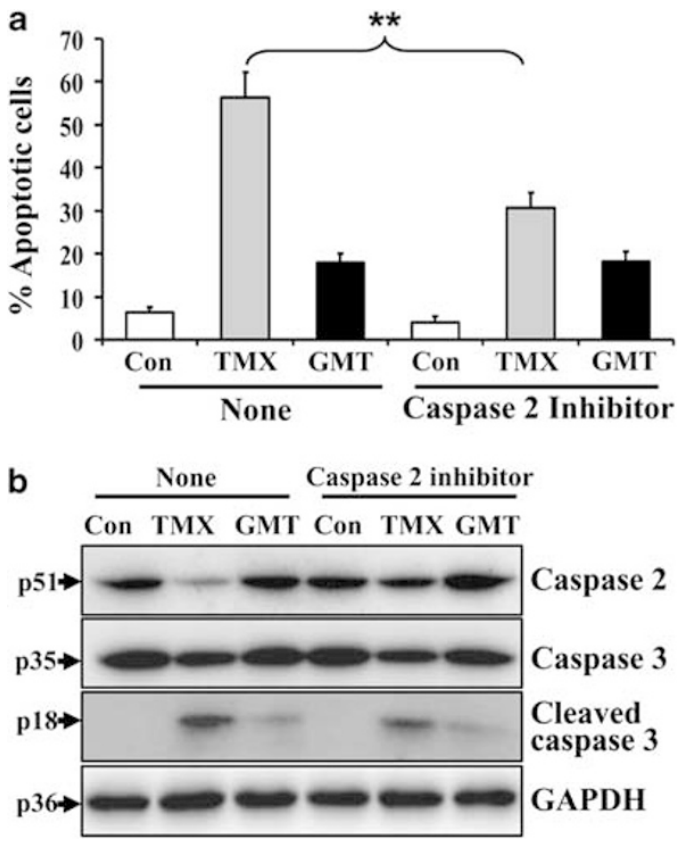

Figure 5 TMX-induced activation of caspase 2 contributes to its apoptotic effects in cholangiocarcinoma cells. Cholangiocarcinoma cells, SK-ChA-1, were pre-incubated with caspase 2 inhibitor for $2 \mathrm{~h}$ and then exposed to TMX $(20 \mu \mathrm{M})$ or GMT $(3 \mu \mathrm{M})$ for $24 \mathrm{~h}$. (a) Apoptosis was determined by Annexin V/propidium iodide staining; (b) the expression/activation of caspases 2 and 3 was determined by western blot analysis. Results shown are mean \pm s.e. $(n=3)$. ${ }^{\star *} P<0.01$ for comparisons. 
that TMX-induced apoptosis is partially dependent on inhibition of pAKT and FLIP expression, as well as activation of caspases $8,10,9$, and $3 .^{10,11,13}$ Hence, we hypothesized that GMT plus TMX would be more effective in inhibiting tumor growth of cholangiocarcinoma in mouse xenografts than either agent alone because of their different mechanisms for inducing apoptosis.

To test our hypothesis, in vitro studies were performed first. Enhanced cell growth inhibition by combination of TMX and GMT was observed, which correlated with increase of apoptosis (Figure 1). There are two major distinct but convergent pathways of apoptosis: the death receptor and mitochondrial pathways. ${ }^{42}$ GMT induces apoptosis in cholangiocarcinoma cells through the mitochondrial pathway (Figure 4a and b). GMT, an analog of deoxycytidine, is a pyrimidine anti-metabolite. The mechanism of action of GMT has been well characterized. GMT is deaminated and inactivated by deoxycytidine deaminase to difluorodeoxyuridine; or else it is activated by deoxycytidine kinase to dFdCMP, dFdCDP, and dFdCTP. The latter are incorporated into DNA, resulting in chain termination. ${ }^{43}$ So its effect is directly against DNA synthesis resulting in apoptosis. ${ }^{26,27}$ In cholangiocarcinoma cells, GMT induces mitochondrial permeabilization with release of cytochrome $c$, which results in the activation of caspase 9. Caspase 9 then ultimately activates caspase 3 and induces apoptosis. ${ }^{42}$

Activation of caspase 8 was also observed in cells stimulated by GMT, suggesting that GMT also activated the extrinsic pathway (Figure 4a). In the extrinsic pathway, ligation of death receptors recruits the adaptor protein Fas-associated death domain (FADD). FADD in turn recruits caspase 8 , which ultimately activates caspase 3 , the key 'executioner' caspase. ${ }^{42}$ Activation of caspase 8 by GMT was also demonstrated in an FADD-independent manner in non-small cell lung cancer cells and pancreatic cancer cell. $^{28,44,45}$ A dominant negative FADD mutant lacking its DED did not interfere with GMT-induced apoptosis. ${ }^{28,44,45}$ Hence, GMT is believed to act primarily through the mitochondria and to trigger apoptosis by an initial release of cytochrome c. ${ }^{28}$ Activation of caspase 8 by GMT may be through an alternative mechanism independent of death receptors. For instance, intracellular glutathione levels and the tyrosine kinase LCK have been postulated to regulate caspase 8 activation independent of extrinsic pathway. ${ }^{28,46,47}$

Our previous data also showed that caspase 8 was partially activated by TMX, but the cleaved active fragment (p18) was not significantly elevated. ${ }^{10,11}$ As a calmodulin antagonist, TMX-induced apoptosis is also partially dependent on inhibition of pAKT and expression of FLIP. ${ }^{11}$ Compared with GMT, the effects of TMX on cytochrome $c$ release and caspase 9 activation were also much weaker (Figure 4a). Their similar effects on the downstream caspase, caspase 3 (Figure 4a), supported the existence of another signaling pathway leading to caspase 3 cleavage and apoptosis. To check this hypothesis, caspase 2 was analyzed as a multifunctional caspase. ${ }^{38-40}$ Caspase 2 is the most conserved caspase across species. Biochemical analysis supports a role of caspase 2 as an initiator caspase, but many contradictory findings render the exact function of this enzyme unresolved. ${ }^{40}$ Caspase 2 has been reported to be involved in DNA damage response, cell-cycle regulation and tumor suppression, although many reports are contradictory and controversial. ${ }^{40,48}$ Caspase 2 appeared to act either upstream or downstream of other caspases depending on the cell type and specific mode investigated. ${ }^{40,49-53}$ Interestingly, caspase 2-mediated cell death was even reported to occur in the absence of any detectable release of mitochondrial factors or even caspase 3 activation, which places caspase 2 independent of both the mitochondrial and death receptor pathways. ${ }^{38} \mathrm{In}$ cholangiocarcinoma cells, TMX was shown to activate caspase 2, whereas GMT had no effect (Figure 4a). Inhibition of caspase 2 activation partially blocked TMX-induced but not GMT-induced activation of caspase 3, which indicates that caspase 2 acts upstream of caspase 3 in cholangiocarcinoma cells (Figure $5 \mathrm{a}$ and $\mathrm{b}$ ). Similarly, in vivo data showed activation of caspase 2 only in TMX-treated tumors. It is reasonable to conclude that the enhanced effect of TMX on GMT-induced apoptosis and tumor inhibition is at least partially mediated by activation of caspase 2. Accordingly, the present study raised many new questions such as how TMX activates caspase 2 and how caspase 2 affects caspase 3 in cholangiocarcinoma cells, which will require further investigations.

In addition to its apoptosis-inducing effects, TMX or GMT has previously been shown to have anti-proliferation effects in some cancer cells, including ovarian, breast, and nonsmall-lung cancer cells. ${ }^{54-59}$ However, we did not find

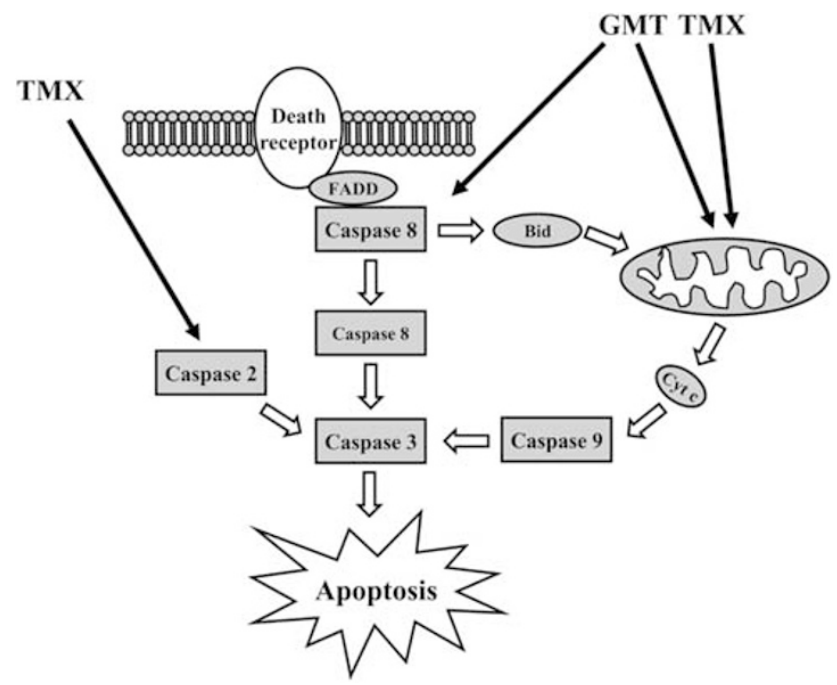

Figure 6 Proposed model of TMX and GMT-induced apoptosis. A model depicts the apoptotic signaling affected by TMX and GMT in cholangiocarcinoma cells. Both TMX and GMT induce release of cytochrome c and activate caspase 9, which ultimately activate caspase 3 and induce apoptosis. GMT also activates caspase 8 . In contrast, TMX activates caspase 2 leading to caspase 3 cleavage, whereas GMT has no effect. 
significant inhibitory effect of GMT, at the concentrations used, on cholangiocarcinoma cell proliferation in vitro or in the mouse xenograft model (Supplementary Figure 1). The combination of GMT and TMX exhibited moderate inhibition on cell proliferation, which was due to the inhibitory effect of TMX on cell proliferation. Nevertheless, the enhancement of TMX on GMT-induced cell death, as well as the efficacy of GMT on tumorigenesis, was largely attributed to the apoptosis-inducing effect of TMX (126\% increased in vitro and $122 \%$ increased in vivo compared with GMT alone).

The present study determined the efficacy of the combination therapy of TMX and GMT on human cholangiocarcinoma tumorigenesis in mouse xenograft model and the responsible molecular mechanisms. TMX and GMT induce apoptosis and inhibit cholangiocarcinoma tumorigenesis. TMX and GMT induce activation of different apoptosis signals in isolated cholangiocarcinoma cells and cholangiocarcinoma tumors in mice. As shown in the proposed model (Figure 6), both TMX and GMT induce the release of cytochrome c and activate caspase 9, which ultimately activate caspase 3 and induce apoptosis. GMT also activates caspase 8 independent on death receptors. In contrast, TMX activates caspase 2 leading to caspase 3 cleavage, whereas GMT has no effect. This is the first report that TMX induces apoptosis by activating caspase 2, which results in its enhanced effects on GMT-induced apoptosis and tumor inhibition. Our studies provide in vivo evidence and mechanistic molecular insight to support the use of TMX and GMT as an effective combination therapy for cholangiocarcinoma.

Supplementary Information accompanies the paper on the Laboratory Investigation website (http://www.laboratoryinvestigation.org)

\section{ACKNOWLEDGEMENT}

This work was supported by VA Merit Review Award (JMM)

\section{DISCLOSURE/CONFLICT OF INTEREST}

The authors declare no conflict of interest.

1. Pitt HA, Dooley WC, Yeo CJ, et al. Malignancies of the biliary tree. Curr Probl Surg 1995;32:1-90.

2. Braconi $C$, Patel T. Cholangiocarcinoma: new insights into disease pathogenesis and biology. Infect Dis Clin North Am 2010;24:871-884.

3. Fava G, Marzioni $M$, Benedetti $A$, et al. Molecular pathology of biliary tract cancers. Cancer Lett 2007;250:155-167.

4. Shaib YH, Davila JA, McGlynn K, et al. Rising incidence of intrahepatic cholangiocarcinoma in the United States: a true increase? J Hepato 2004;40:472-477.

5. Khan SA, Thomas HC, Davidson BR, et al. Cholangiocarcinoma. Lancet 2005;366:1303-1314.

6. Mosconi S, Beretta GD, Labianca R, et al. Cholangiocarcinoma. Crit Rev Oncol Hematol 2009;69:259-270.

7. Lazaridis KN, Gores GJ. Cholangiocarcinoma. Gastroenterology 2005;128:655-667.

8. Patel T. Increasing incidence and mortality of primary intrahepatic cholangiocarcinoma in the United States. Hepatology 2001;33: 1353-1357.

9. Cohen GM. Caspases: the executioners of apoptosis. Biochem J 1997;326:1-16.

10. Ahn EY, Pan G, Oh JH, et al. The combination of calmodulin antagonists and interferon- $\gamma$ induces apoptosis through caspase- dependent and -independent pathways in cholangiocarcinoma cells. Am J Pathol 2003;163:2053-2063.

11. Pawar $\mathrm{P}, \mathrm{Ma} \mathrm{L}$, Byon $\mathrm{CH}$, et al. Molecular mechanisms of tamoxifen therapy for cholangiocarcinoma: role of calmodulin. Clin Cancer Res 2009;15:1288-1296.

12. Pan G, Vickers $S M$, Pickens $A$, et al. Apoptosis and tumorigenesis in human cholangiocarcinoma cells. Involvement of Fas/APO-1 (CD95) and calmodulin. Am J Pathol 1999;155:193-203.

13. Vickers SM, Jhala NC, Ahn EY, et al. Tamoxifen (TMX)/Fas induced growth inhibition of human cholangiocarcinoma (HCC) by $g$ interferon (IFN-g). Ann Surg 2002;235:872-878.

14. Ahn EY, Pan G, Vickers SM, et al. IFN-gamma upregulates apoptosisrelated molecules and enhances Fas-mediated apoptosis in human cholangiocarcinoma. Int J Cancer 2002;100:445-451.

15. Que FG, Phan VA, Phan VH, et al. Cholangiocarcinomas express Fas ligand and disable the Fas receptor. Hepatology 1999;30:1398-1404.

16. Chen $\mathrm{Y}, \mathrm{Xu} \mathrm{J}$, Jhala $\mathrm{N}$, et al. Fas-mediated apoptosis in cholangiocarcinoma cells is enhanced by $3,3^{\prime}$-diindolylmethane through inhibition of AKT signaling and FLICE-like inhibitory protein. Am J Pathol 2006;169:1833-1842.

17. Cole MP, Jones CT, Todd ID. A new anti-oestrogenic agent in late breast cancer. An early clinical appraisal of $\mathrm{ICl} 46474$. Br J Cancer 1971;25:270-275.

18. Ward HW. Anti-oestrogen therapy for breast cancer: a trial of tamoxifen at two dose levels. Br Med J 1973;1:13-14.

19. Mandlekar S, Kong AN. Mechanisms of tamoxifen-induced apoptosis. Apoptosis 2001;6:469-477.

20. Kubicka S, Rudolph KL, Tietze MK, et al. Phase II study of systemic gemcitabine chemotherapy for advanced unresectable hepatobiliary carcinomas. Hepatogastroenterology 2001;48:783-789.

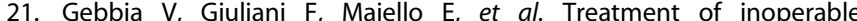
and/or metastatic biliary tree carcinomas with single-agent gemcitabine or in combination with levofolinic acid and infusional fluorouracil: results of a multicenter phase II study. J Clin Oncol 2001; 19:4089-4091.

22. Alberts SR, Al-Khatib H, Mahoney MR, et al. Gemcitabine, 5-fluorouracil, and leucovorin in advanced biliary tract and gallbladder carcinoma: a North Central Cancer Treatment Group phase II trial. Cancer 2005;103:111-118.

23. Knox JJ, Hedley D, Oza A, et al. Combining gemcitabine and capecitabine in patients with advanced biliary cancer: a phase II trial. $\mathrm{J}$ Clin Oncol 2005;23:2332-2338.

24. Mazhar D, Stebbing J, Bower M. Chemotherapy for advanced cholangiocarcinoma: what is standard treatment? Future Oncol 2006;2:509-514.

25. André $T$, Tournigand $C$, Rosmorduc $O$, et al. Gemcitabine combined with oxaliplatin (GEMOX) in advanced biliary tract adenocarcinoma: a GERCOR Study. Ann Oncol 2004;15:1339-1343.

26. Nabhan C, Gajria D, Krett NL, et al. Caspase activation is required for gemcitabine activity in multiple myeloma cell lines. Mol Cancer Ther 2002;1:1221-1227.

27. Huang $\mathrm{P}$, Chubb $\mathrm{S}$, Hertel LW, et al. Action of $2^{\prime}, 2^{\prime}$-difluorodeoxycytidine on DNA synthesis. Cancer Res 1991;51:6110-6117.

28. Christgen M, Schniewind B, Jueschke A, et al. Gemcitabine-mediated apoptosis is associated with increased CD95 surface expression but is not inhibited by DN-FADD in Colo357 pancreatic cancer cells. Cancer Lett 2005;227:193-200.

29. Yu WD, Ma Y, Flynn G, et al. Calcitriol enhances gemcitabine anti-tumor activity in vitro and in vivo by promoting apoptosis in a human pancreatic carcinoma model system. Cell Cycle 2010;15: 3022-3029.

30. Hastak K, Alli E, Ford JM. Synergistic chemosensitivity of triple-negative breast cancer cell lines to PARP inhibition, gemcitabine and cisplatin. Cancer Res 2010;20:7970-7980.

31. da Silva GN, de Castro Marcondes JP, de Camargo EA, et al. Cell cycle arrest and apoptosis in TP53 subtypes of bladder carcinoma cell lines treated with cisplatin and gemcitabine. Exp Biol Med (Maywood) 2010;235:814-824

32. Hall FM. Cisplatin plus gemcitabine for biliary tract cancer. $N$ Engl $J$ Med 2010;363:192-193.

33. Galvão FH, Pestana JO, Capelozzi VL. Fatal gemcitabine-induced pulmonary toxicity in metastatic gallbladder adenocarcinoma. Cancer Chemother Pharmacol 2010;65:607-610. 
34. Ko E, Lee S, Goodman A. Gemcitabine pulmonary toxicity in ovarian cancer. Oncologist 2008;13:807-881.

35. Shaib W, Lansigan F, Cornfeld D, et al. Gemcitabine-induced pulmonary toxicity during adjuvant therapy in a patient with pancreatic cancer. JOP 2008;9:708-714.

36. Barlési $F$, Villani $P$, Doddoli $C$, et al. Gemcitabine-induced severe pulmonary toxicity. Fundam Clin Pharmacol 2004;18:85-91.

37. Ustundag Y, Bronk SF, Gores GJ. Proteasome inhibition-induces endoplasmic reticulum dysfunction and cell death of human cholangiocarcinoma cells. World J Gastroenterol 2007;13:851-857.

38. Sidi S, Sanda T, Kennedy RD, et al. Chk1 suppresses a caspase-2 apoptotic response to DNA damage that bypasses p53, $\mathrm{Bcl}-2$, and caspase-3. Cell 2008;133:864-877.

39. Dahal GR, Karki P, Thapa A, et al. Caspase-2 cleaves DNA fragmentation factor (DFF45)/inhibitor of caspase-activated DNase (ICAD). Arch Biochem Biophys 2007;468:134-139.

40. Krumschnabel G, Sohm B, Bock F, et al. The enigma of caspase-2: the laymen's view. Cell Death Differ 2009;16:195-207.

41. Patel T. Cholangiocarcinoma. Nat Clin Pract Gastroenterol Hepatol 2006;3:33-42.

42. Hotchkiss RS, Strasser A, McDunn JE, et al. Cell death. N Engl J Med 2009;361:1570-1583.

43. Sandler A, Ettinger DS. Gemcitabine: single-agent and combination therapy in non-small cell lung cancer. Oncologist 1999;4:241-251.

44. Ferreira CG, Span SW, Peters GJ, et al. Chemotherapy triggers apoptosis in a caspase-8-dependent and mitochondria-controlled manner in the nonsmall cell lung cancer cell line $\mathrm{NCl}-\mathrm{H} 460$. Cancer Res 2000;60:7133-7141.

45. Bandala E, Espinosa M, Maldonado V, et al. Inhibitor of apoptosis-1 (IAP-1) expression and apoptosis in non-small-cell lung cancer cells exposed to gemcitabine. Biochem Pharmacol 2001;62:13-19.

46. Kitamura K, Minami Y, Yamamoto K, et al. Involvement of CD95independent caspase 8 activation in arsenic trioxide-induced apoptosis. Leukemia 2000;14:1743-1750.

47. Belka C, Marini $P$, Lepple-Wienhues $A$, et al. The tyrosine kinase Ick is required for CD95-independent caspase-8 activation and apoptosis in response to ionizing radiation. Oncogene 1999;18:4983-4992.
48. Kumar S. Caspase 2 in apoptosis, the DNA damage response and tumour suppression: enigma no more? Nat Rev Cancer 2009;9:897-903.

49. Lassus $P$, Opitz-Araya $X$, Lazebnik $Y$. Requirement for caspase- 2 in stress-induced apoptosis before mitochondrial permeabilization. Science 2002;297:1352-1354.

50. Lin CF, Chen CL, Chang WT, et al. Sequential caspase- 2 and caspase- 8 activation upstream of mitochondria during ceramide and etoposideinduced apoptosis. J Biol Chem 2004;279:40755-40761.

51. Lin $\mathrm{CF}$, Chen $\mathrm{CL}$, Chang $\mathrm{WT}$, et al. $\mathrm{Bcl}-2$ rescues ceramide- and etoposide-induced mitochondrial apoptosis through blockage of caspase-2 activation. J Biol Chem 2005;280:23758-23765.

52. Samraj AK, Sohn D, Schulze-Osthoff K, et al. Loss of caspase-9 reveals its essential role for caspase-2 activation and mitochondrial membrane depolarization. Mol Biol Cell 2007;18:84-93.

53. Werner AB, Tait SW, de Vries E, et al. Requirement for aspartate-cleaved bid in apoptosis signaling by DNA-damaging anti-cancer regimens. J Biol Chem 2004;279:28771-28780.

54. McNamara DA, Harmey J, Wang JH, et al. Tamoxifen inhibits endothelia cell proliferation and attenuates VEGF-mediated angiogenesis and migration in vivo. Eur J Surg Oncol 2001;27:714-718.

55. Duffy SM, Lawley WJ, Kaur D, et al. Inhibition of human mast cell proliferation and survival by tamoxifen in association with ion channel modulation. J Allergy Clin Immunol 2003;112:965-972.

56. Mabuchi S, Ohmichi M, Kimura A, et al. Tamoxifen inhibits cell proliferation via mitogen-activated protein kinase cascades in human ovarian cancer cell lines in a manner not dependent on the expression of estrogen receptor or the sensitivity to cisplatin. Endocrinology 2004; 145:1302-1313.

57. Jones JL, Daley BJ, Enderson BL, et al. Genistein inhibits tamoxifen effects on cell proliferation and cell cycle arrest in T47D breast cancer cells. Am Surg 2002;68:575-577.

58. Reddel RR, Sutherland RL. Tamoxifen stimulation of human breast cancer cell proliferation in vitro: a possible model for tamoxifen tumour flare. Eur J Cancer Clin Oncol 1984;20:1419-1424.

59. Pace $E$, Melis $M$, Siena $L$, et al. Effects of gemcitabine on cell proliferation and apoptosis in non-small-cell lung cancer (NSCLC) cell lines. Cancer Chemother Pharmacol 2000;46:467-476. 